\title{
3D Registration of the Point Cloud Data Using Parameter Adaptive Super4PCS Algorithm in Medical Image Analysis
}

\author{
Shun Su \\ the State Key Laboratory of Robotics, \\ Shenyang Institute of Automation, \\ Chinese Academy of Sciences, \\ Shenyang 110016, China; the \\ Institutes for Robotics and Intelligent \\ Manufacturing, Chinese Academy of \\ Sciences, Shenyang 110016, China; \\ School of Computer Science and \\ Technology, University of Chinese \\ Academy of Sciences, Beijing 100049, \\ China \\ Sushun@Sia.Cn
}

\author{
Guoli Song* \\ the State Key Laboratory of Robotics, \\ Shenyang Institute of Automation, \\ Chinese Academy of Sciences, \\ Shenyang 110016, China; the \\ Institutes for Robotics and Intelligent \\ Manufacturing, Chinese Academy of \\ Sciences, Shenyang 110016, China \\ songgl@sia.cn
}

\author{
Yiwen Zhao \\ the State Key Laboratory of Robotics, \\ Shenyang Institute of Automation, \\ Chinese Academy of Sciences, \\ Shenyang 110016, China; the \\ Institutes for Robotics and Intelligent \\ Manufacturing, Chinese Academy of \\ Sciences, Shenyang 110016, China \\ songgl@sia.cn
}

\begin{abstract}
In this article, we use the parameter-adaptive Super4PCS algorithm to achieve high-precision registration of medical point clouds. First, generate the corresponding point cloud from the biological data (CT, MRI) to be registered. Then analyze the characteristics of the point cloud to be registered, and use it to adaptively set the parameters of Super4PCS, and finally perform point cloud registration. We compare the performance of six different algorithms with their accuracy and robustness. The accuracy, robustness of our method are the best. At the same time, no parameter input is required which is very convenient for medical workers. Experiments on medical models demonstrate the efficiency and robustness of our algorithm.
\end{abstract}

\section{CCS CONCEPTS}

- Human-centered computing;

\section{KEYWORDS}

Medical image registration, point cloud registration

\section{ACM Reference Format:}

Shun Su, Guoli Song, and Yiwen Zhao. 2021. 3D Registration of the Point Cloud Data Using Parameter Adaptive Super4PCS Algorithm in Medical Image Analysis. In 2021 4th International Conference on Digital Medicine and Image Processing (DMIP '21), November 12-15, 2021, Kyoto, fapan. ACM, New York, NY, USA, 6 pages. https://doi.org/10.1145/3506651.3506652

${ }^{*}$ Corresponding author.

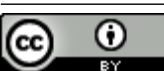

This work is licensed under a Creative Commons Attribution International 4.0 License.

DMIP '21, November 12-15, 2021, Kyoto, Japan

(c) 2021 Copyright held by the owner/author(s).

ACM ISBN 978-1-4503-8648-7/21/11.

https://doi.org/10.1145/3506651.3506652

\section{INTRODUCTION}

Point cloud registration is a process of finding rigidity transformation of two point cloud [1]. It can achieve medical image registration by registering the preoperative point cloud generated by multimodal medical images or combination the preoperative point cloud with the intraoperative point cloud obtained by different data acquisition equipment. Various point cloud registration methods are widely used in medical image fusion or registration. In 2017, Fan et al. [2] used the G-ICP point cloud registration algorithm [3] to perform point cloud registration of the nervous system. This method solves the problem of brain deformation in neurosurgery and achieves point cloud registration of preoperative and intraoperative brains with millimeter accuracy. In 2018, Wang et al. [4] combined point cloud registration technology with ultrasoundbased surgical navigation to achieve a non-invasive, non-radiative image navigation system.

Our proposed point cloud registration method takes the neurosurgery image navigation as the background. First, Generate the point cloud of medical image to be registered. Using preoperative MRI and CT images, various intracranial anatomical structures are generated based on different threshold windows, and the complete point cloud model A to be registered is generated. In terms of preoperative and intraoperative registration, using the intraoperative Kinect II device [5] to scan the exposed part of the patient's head, a partial point cloud model B to be registered is generated. Our main contributions are:

1. An original multisystem calibration method was proposed, and a solution for calibration between multiple systems was provided.

2. A fully automatic registration method for medical image point cloud is proposed based on improved Super4PCS [6] and ICP [7] point cloud registration.

3. A variety of existing point cloud registration methods were tested on medical point cloud registration. 


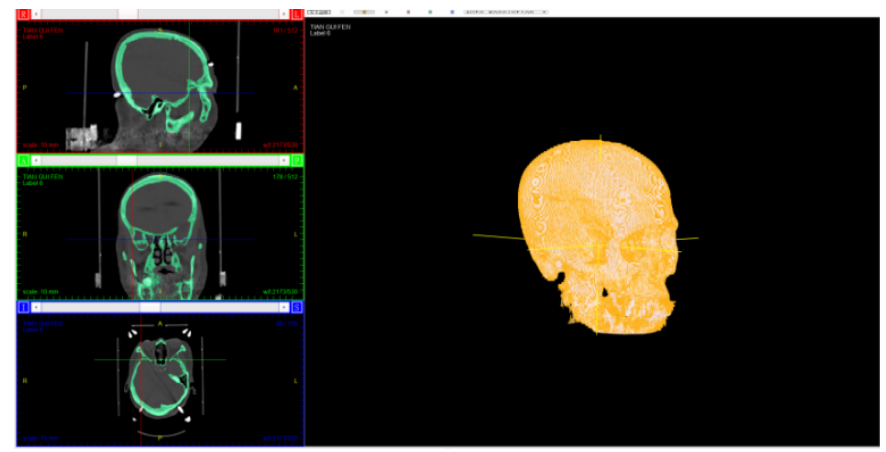

Figure 1: Software interface used to generate the preoperative models

\section{MATERIALS AND METHODS}

\subsection{System software and hardware components}

The hardware devices used in this system consisted of a workstation (ThinkPad P51) with a 64-bit Intel ${ }^{\circledR}$ Core $^{\text {TM }}$ i7 7700HQ CPU, 16 GB of memory, the Microsoft Windows 10 operating system and a Kinect II. The Kinect II is a line of motion sensing input device produced by Microsoft. It has a depth camera and a color camera which can be used to capture scans that can be utilized to obtain the model's point cloud.

\subsection{Modeling from medical images}

In our experiment, the preoperative models were generated by our own software based on different threshold windows. Prior to this, we imported the CT or MRI images into the software and converted their formats from .dicom to either .txt or .obj. The software interface is shown in Figure 1. The intraoperative model was collected by the Kinect and treated directly with Kinect SDK.

\subsection{Point cloud registration}

Currently, there are many point cloud registration algorithms, ranging from the initial ICP algorithm to the later CPD algorithm [8] to 4PCS [9] and Super4PCS [6]. Our proposed navigation system is used in situations where the point cloud collected during the surgical procedure has low overlap with the point cloud obtained prior to the procedure. After experimental testing and analysis, the Super4PCS point cloud registration algorithm was selected.

The Super4PCS algorithm, which is based on the 4PCS algorithm, performs point cloud registration. The basic idea originates from the RANSAC algorithm [10], although the strategy for determining the corresponding point pairs has been optimized, and the original randomly selected three different points have been modified to stem from the four coplanar points in the source point cloud, and are determined in the target point cloud. The four points form four sets of corresponding points that can enhance the robustness of the algorithm to some extent. The theoretical basis of the four-point uniform set algorithm is the affine invariance of coplanar four-point pairs. In the affine transformation, the ratio $\mathrm{r}=\left\|\frac{a-b}{a-c}\right\|$ determined by the three defined coplanar points $\mathrm{a}, \mathrm{b}$, and $\mathrm{c}$ is constant. Then, for a given non-collinear coplanar four points $a, b, c$, and d, where $\mathrm{ab}$ and cd intersect at e, the following two ratios $r_{1}=\left\|\frac{a-\mathrm{e}}{a-b}\right\|, \mathrm{r}_{2}=$ $\left\|\frac{c-\mathrm{e}}{c-d}\right\|$ can be determined. $r_{1}$ and $r_{2}$ are then also invariant in the affine transformation, i.e., $r_{1}$ and $r_{2}$ determined by the four coplanar points in the source point cloud while $r_{1}$ and $r_{2}$ determined by the corresponding four points in the target point cloud are identical.

The principle of determining corresponding pairs of points based on affine invariance can be described as follows: Assuming a set of four coplanar points in a given source point cloud $\mathrm{P}$, the ratios $r_{1}$ and $r_{2}$ determined by the four points are available, as shown in Figure 2

For any point pair $q_{1}$ and $q_{2}$ in the target point cloud Q, the possible intersection points $e_{1}$ and $e_{2}$ are calculated:

$$
\mathrm{e}_{1}=\mathrm{q}_{1}+r_{1}\left(q_{2}-q_{1}\right), e_{2}=\mathrm{q}_{1}+r_{2}\left(q_{2}-q_{1}\right)
$$

If there are two pairs of such points in $\mathrm{Q}$ such that the $e_{1}$ of the pair and the $e_{2}$ of the other pair are equal within the error tolerance, then the two pairs can be considered as the corresponding coplanar points of the base given in P, as shown in Figure 7, in which the gray dots represent $e_{1}$ and the yellow dots represent $e_{2}$. The points $q_{1}, q_{2}, q_{3}$, and $q_{4}$ then correspond to a, b, c, and $\mathrm{d}$

Given a source point cloud $\mathrm{P}$, a target point cloud $\mathrm{Q}$, a distance uncertainty metric $\epsilon$, and an estimated overlap rate f of two sections of the point clouds, the basic principles of the Super4PCS algorithm are as follows:

Select coplanar four points from $\mathrm{P}$ to form a base (represented by $\mathrm{B}$ ). The base time is chosen to follow the principle of distance maximization, i.e., to ensure that the distance between the points is large but does not exceed a certain threshold. This threshold can be determined by the overlap rate $f$. The three-plus-one strategy is adopted when selecting points, i.e., three different points are first selected on the surface of the point cloud which may be overlapping regions, and the fourth point is then determined by the principle of coplanar non-collinearity. After determining the base $B \equiv\left\{\mathrm{p}_{a}, \mathrm{p}_{b}, \mathrm{p}_{c}, \mathrm{p}_{d}\right\}$, the corresponding ratios $\mathrm{r}_{1}$ and $\mathrm{r}_{2}$ can be obtained, as well as two dot pitches. The sets $S_{1}$ and $S_{2}$ in $Q$ are determined as follows:

$$
\begin{aligned}
& \mathrm{S}_{1} \equiv\left\{\left(\mathrm{q}_{i}, \mathrm{q}_{j}\right) \mid \mathrm{q}_{i}, \mathrm{q}_{j} \in Q,\left\|p_{i}-p_{j}\right\| \in\left[d_{1}-\varepsilon, d_{1}+\varepsilon\right]\right\} \\
& \mathrm{S}_{2} \equiv\left\{\left(\mathrm{q}_{i}, \mathrm{q}_{j}\right) \mid \mathrm{q}_{i}, \mathrm{q}_{j} \in Q,\left\|p_{i}-p_{j}\right\| \in\left[d_{2}-\varepsilon, d_{2}+\varepsilon\right]\right\}
\end{aligned}
$$

For each point $\mathrm{q}_{\mathrm{i}}$ in $\mathrm{Q}$, with each point as the center of a sphere, draw spheres with radii $\mathrm{R}=\mathrm{d}_{1}$ and $\mathrm{R}=\mathrm{d}_{2}$, respectively, such that the points $\mathrm{q}_{\mathrm{i}}$ satisfy the condition of $S_{1}$, with those points falling within the range $\left[d_{1}-\varepsilon, d_{1}+\varepsilon\right]$, and the points $\mathrm{q}_{j}$ satisfy the condition of $S_{2}$, with those points falling within the range $\left[d_{1}-\varepsilon, d_{1}+\varepsilon\right]$.

The four-point set corresponding to the base is extracted in $Q$ and the incorrect four-point set is eliminated. As shown in Figure 3 , for the given base $\mathrm{B}$ in $\mathrm{P}$, there is a corresponding point set $\left\{\mathrm{q}_{a}, q_{b}, q_{c}, q_{d}\right\}$ in $\mathrm{Q}$, but there may be an incorrect corresponding point set $\left\{\mathrm{q}_{a}, q_{b}, q_{c}{ }^{\prime}, q_{d}{ }^{\prime}\right\}$. The traditional $4 \mathrm{PCS}$ algorithm takes more time to propose the incorrect corresponding point set, whereas the Super4PCS algorithm can remove the incorrect point set while extracting the four-point set, i.e., it can obtain the unique four-point set corresponding to the base, thereby accelerating the algorithm.

As can be seen from Figure 3, if a given base $\left\langle\mathrm{d}_{1}, \mathrm{~d}_{2}, r_{1}, r_{2}, \theta\right\rangle$ is available, a unique corresponding 4-point set can be determined in the target point cloud. 

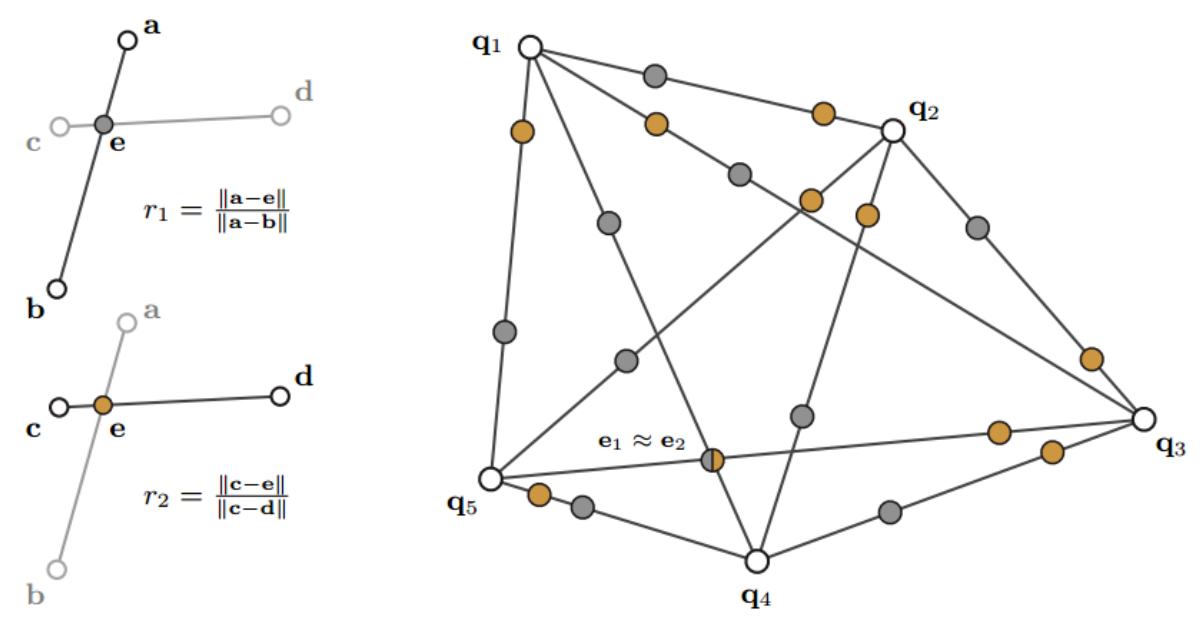

Figure 2: Affine transformation diagram
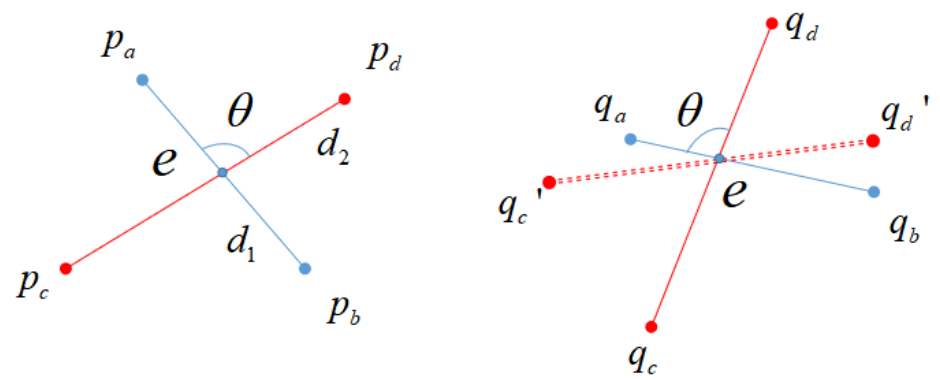

Figure 3: Mismatched point pair

From the corresponding point sets $\left\{p_{a}, p_{b}, p_{c}, p_{d}\right\}$ and $\left\{\mathrm{q}_{a}, q_{b}, q_{c}{ }^{\prime}, q_{d}{ }^{\prime}\right\}$ the rigid body transformation matrix $\mathrm{T}$ is calculated. The source point cloud $\mathrm{P}$ is transformed using $\mathrm{T}$, and the number of points where the statistically transformed point cloud and the nearest point in the target point cloud are less than a certain threshold are used to represent the quality of the transformation matrix T. We then iterate through the above 4 steps until the optimal $\mathrm{T}$ is found such that that the 2-point clouds are close enough.

In terms of medical image registration, the point cloud model obtained by preoperative MRI and CT acquisition has higher resolution of point cloud information than the intraoperative scanning device, and the detail preservation effect is better. The original Super 4PCS algorithm needs to set six parameters to achieve registration. Such as Overlap, delta, n_points, norm_diff, max_color_diff and max_time_seconds. Among them, when different parameter values are set for A and B, the effect is obviously different. Other four parameters could be set fixed values for medical image registration.
Overlap is the coverage of two pending point clouds. Because medical image registration does not interfere with other nonregistered objects, and the scale is consistent. We can approximate the overlap by comparing the number of point clouds with the resolution of the point cloud to be registered. The resolution res is calculated as follows, $\mathrm{P}$ is the point cloud to calculate the resolution.

$$
\text { res }=\frac{\sum_{i=1}^{n} \sum_{j=1}^{n} \min \left\|P_{i}-P_{j}\right\|}{n}
$$

By sampling the number of point clouds with fixed steps (every small cube in the Figure 4) the point cloud to be registered is reduced to the same resolution, and the overlap is estimated by calculating the ratio of the number of point clouds, and the experimental results are better.

Delta is a concept in random sample consistency that is used to set the range of fit thresholds. After testing, the delta value can be set according to the pre-operative high-resolution point cloud acquired by MRI or CT. Since the point cloud density distribution obtained by MRI or CT is uniform, the delta value is approximated 
Table 1: The registration result of different method on customs dataset

\begin{tabular}{lllllll}
\hline $\begin{array}{l}\text { Point cloud registration } \\
\text { method }\end{array}$ & PCA + KdTree + & FPFH + Ransac + & CPD(rigid) & ICP & 4PCS + ICP & Super4PCS + ICP \\
\hline Run Time/s & ICP & ICP & & & \\
RMS & 112 & 135 & 108 & 200 & 80 & 62 \\
\end{tabular}

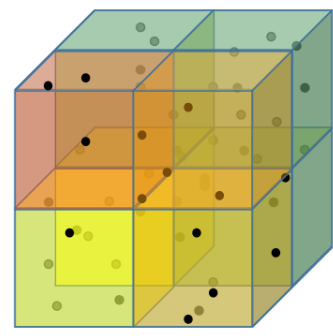

Figure 4: Small cube represent the fix step, black points represent the point clouds. It could reduce the point cloud resolution via this way.

by calculating the point cloud density. By calculating the bounding box volume of the point cloud, the point cloud density is obtained by dividing the point cloud data amount by the bounding box volume.

$$
\text { Delta }=P n / V b
$$

$\boldsymbol{P n}$ is the number of point cloud, $\boldsymbol{V} \boldsymbol{b}$ is the volume of the bounding box.

Experiments have shown that this value is better for highresolution point clouds obtained by medical imaging equipment such as MRI and CT. The other four parameters can be used for medical image registration norm_diff and max_color_diff, and the color information of MRI and CT cannot be used. When n_points and max_time_seconds are not considered for registration time, the larger the value, the more accurate the registration effect is. Considering that pre-medical planning does not care about the registration time, the $n \_$points value is set to the number of intrasampling point clouds only if the registration accuracy is concerned. Max_time_seconds is set to 100 .

We then add the ICP algorithm as the final step of optimization processing. After the Super4PCS algorithm is used for the coarse registration, the ICP algorithm is used for the fine registration. This procedure can greatly reduce the number of iterations compared to utilizing the ICP algorithm on its own, thereby reducing processing time, and resulting in better registration.

\section{EXPERIMENTS AND RESULTS}

\subsection{Comparison of different methods on a standard dataset}

Considering the accuracy requirements of medical image registration, we tested several mainstream traditional point cloud registration algorithms. The Stanford bunny 3D test model [11], developed

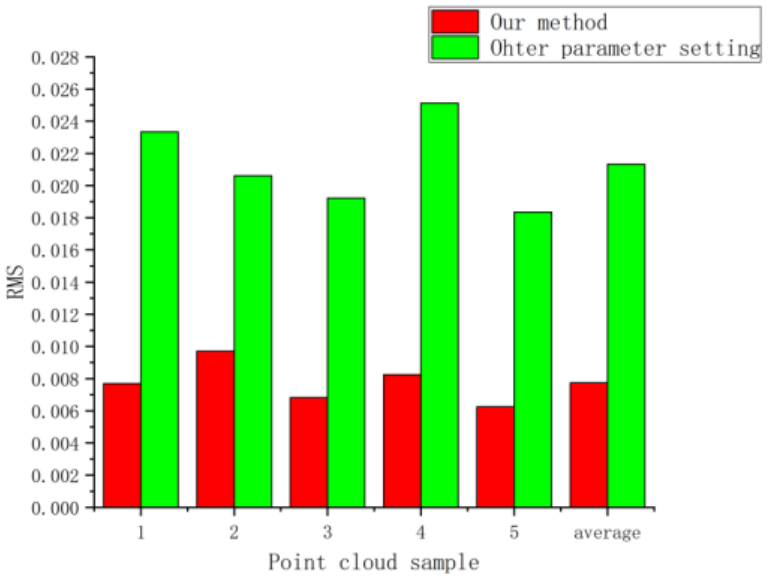

Figure 5: RMS of point cloud registration with different parameter values

by Turk and Levoy in 1994, was used as test data. The results are shown in Table 1. The Root-Mean-Square (RMS) error is used as an evaluation.

The six-point cloud registration algorithms exhibited little difference in performance when using the Stanford bunny standard point cloud as test data. The registration times only varied slightly. Due to the large number of iterations and the large amount of calculation, the ICP took relatively long compared to the other algorithms. Owing to the small number of point clouds in the bunny dataset, the efficiency problem is slightly obscured. When faced with a large number of point clouds in a medical image requiring registration with a point cloud, the efficiency problem may be magnified extremely.

In a medical context, the system needs to deal with the registration of physiological tissue structures. The ICP algorithm was found to be both time-consuming and prone to mismatch. As opposed to the ICP class algorithm, the CPD, 4PCS, and Super4PCS algorithms will not fail in cases of low overlap. The CPD algorithm performs slightly better than the ICP, although its stability and efficiency are not as good as those of the 4PCS and Super4PCS algorithms. The effect of the Super4PCS is similar to that of the 4PCS, and its registration speed is slightly faster.

\subsection{Parameter adaptive optimization effect on Super4PCS}

The dataset used for adaptive parameter optimization comparison was a spine model's point cloud obtained by CT and Kinect. 


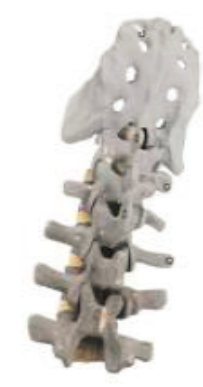

(a)

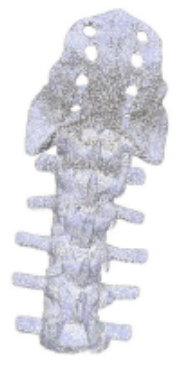

(b)

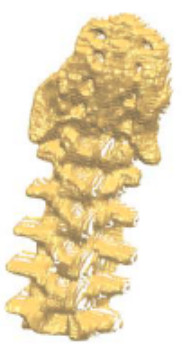

(c)

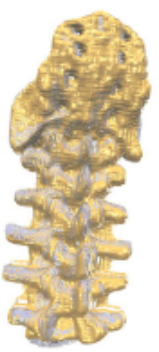

(d)

Figure 6: (a) Medical spine model; (b) CT scan of medical spine model; (c) One-time frontal scan of the Kinect II; (d) Point cloud registration result

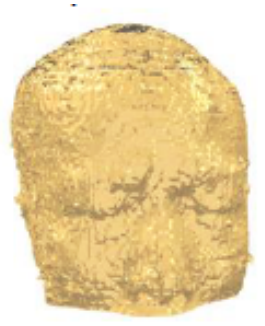

(a)

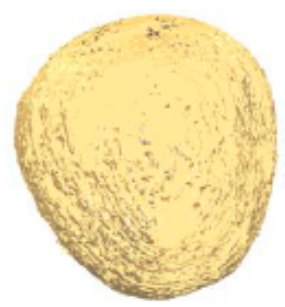

(d)

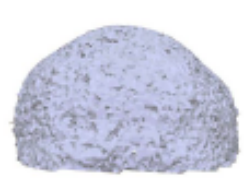

(b)

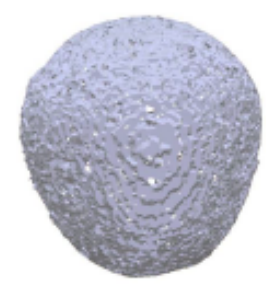

(e)

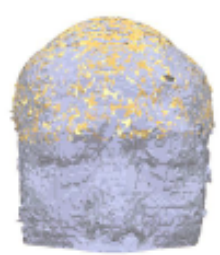

(c)

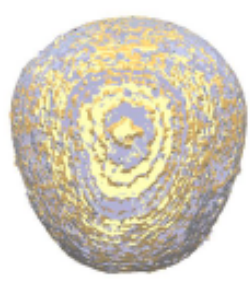

(f)

Figure 7: Registration renderings(a) Main view of preoperative model; (b) Main view of intraoperative model; (c) Main view after registration; (d) Top view of preoperative model; (e) Top view of intraoperative model; (f) Top view after registration

The Table 2 lists the comparison of different parameter settings and our methods.

Figure 5 shows the average RMS of the Super4PCS algorithm after setting our method and other parameter values in multiple sets of different point cloud samples. If the parameter value setting method is deemed to be a registration failure if the RMS exceeds $0.05 \mathrm{~m}$, the result is not counted. The smaller RMS value can reduce the registration time of the subsequent ICP algorithm, so we can figure out that our method can optimize the effect of Super4PCS+ICP in medical image registration.
Figure 6 (a) is the medical spine model. Figure $6(\mathrm{~b})$ is point cloud generated by medical spine model's CT scan. Figure 6 (c) is a point cloud generated by a one-time frontal scan by the Kinect II. Figure 6 (d) is the effect of point cloud registration. The two-point clouds' registration effect is good.

The proposed registration method should be considered for application when only the surface data of the patient can be obtained, but the surgeon requires complete information concerning internal or occluded anatomy. We register by using the Kinect II to scan the patient's head surface for the intraoperative point cloud and 
Table 2: RMS of point cloud registration with different parameter values

\begin{tabular}{lllllll}
\hline $\begin{array}{l}\text { Sample number/ Parameter } \\
\text { number }\end{array}$ & 1 & 2 & 3 & 4 & 5 & ours \\
\hline 1 & 0.00083 & 0.00145 & 0.00128 & 0.00354 & 0.00271 & 0.00097 \\
2 & 0.00119 & 0.00083 & 0.00093 & 0.00112 & 0.00278 & 0.00076 \\
3 & 0.00053 & 0.00067 & 0.00062 & 0.00098 & 0.00101 & 0.00032 \\
\hline
\end{tabular}

Table 3: Registration error of 5 selected point cloud on the skull

\begin{tabular}{lllllll}
\hline Sample number & 1 & 2 & 3 & 4 & 5 & Average \\
\hline Error/m & 0.000611 & 0.000758 & 0.000729 & 0.00112 & 0.000439 & 0.000733 \\
\hline
\end{tabular}

the patient's preoperative MRI generated preoperative point cloud. Point cloud registration effect is shown as Figure 7. We adopted the registration error of five marker points as the evaluation index, as shown in Table 3. Five selected marker points' average translation error is $0.733 \mathrm{~mm}$.

\section{CONCLUSION}

Our method achieves the optimal registration effect of the Super4PCS algorithm. The effect of it is better than other traditional registration methods. It achieves sub-millimeter registration accuracy in medical image registration. In the future, we will focus more on the point cloud registration of ground overlap rate. This will allow the system to be used in more minimally invasive surgical environments, in which it will be registered with a complete point cloud acquired prior to surgery and a point cloud with a lower overlap rate during surgery.

\section{ACKNOWLEDGMENTS}

This research was funded by the National Key R\&D Program of China under grant 2017YFB1303003, the National Natural Science Foundation of China under grants 62073314 and 61821005, Youth Innovation Promotion Association of the Chinese Academy of Sciences under Grant 2019205, Program GQRC-19-20 and the Special
Fund for High-level Talents (Shizhen Zhong Team) of the People's Government of Luzhou Southwestern Medical University.

\section{REFERENCES}

[1] J. Mitra, Niloy \& Gelfand, N \& Pottmann, Helmut \& Guibas, Leonidas. (2004). Registration of Point Cloud Data from a Geometric Optimization Perspective. ACM International Conference Proceeding Series. 71. 23-32. 10.1145/1057432.1057435.

[2] Fan Y, Yao X, Xu X. Automatic Surface-Matching Spatial Registration Method for Neuronavigation Using Globally Optimal Iterative Closest Point [J]. Journal of Medical Imaging \& Health Informatics, 2017, 7(5):1099-1102.

[3] Yang J, Li H, Jia Y. Go-ICP: Solving 3D Registration Efficiently and Globally Optimally [C]// 2013 IEEE International Conference on Computer Vision (ICCV). IEEE Computer Society, 2013.

[4] Wang L et al, "An Automated Calibration Method of Ultrasonic Probe Based on Coherent Point Drift Algorithm," in IEEE Access, vol. 6, pp. 8657-8665, 2018, doi: 10.1109/ACCESS.2018.2791582.

[5] Fankhauser P, Bloesch M, Rodriguez D, et al. Kinect v2 for mobile robot navigation: Evaluation and modeling [C]//Advanced Robotics (ICAR), 2015 International Conference on. IEEE, 2015: 388-394.

[6] Mellado N, Aiger D, Mitra N J. Super4PCS Fast Global Pointcloud Registration via Smart Indexing [J]. Computer Graphics Forum, 2014, 33(5):205-215.

[7] Besl P J, Mckay N D. A method for registration of 3-D shapes [J]. IEEE Trans. Pattern Anal. Mach. Intell, 1992.

[8] Myronenko A, Song X. Point Set Registration: Coherent Point Drift [J]. IEEE Transactions on Pattern Analysis and Machine Intelligence, 2010.

[9] Aiger D, Mitra N J, Cohen-Or D. 4-points congruent sets for robust pairwise surface registration [J]. ACM Transactions on Graphics, 2008, 27(3):1.

[10] Martin A. Fischler \& Robert C. Bolles.Random Sample Consensus: A Paradigm for Model Fitting with Applications to Image Analysis and Automated Cartography.Comm.ACM.24(6):381-395. \%ACM. 24 (6): 381-395.

[11] Curless B, Levoy M. A Volumetric Method for Building Complex Models from Range Images. ACM, 1999. 